\title{
Association of RB/p16-Pathway Perturbations with DCIS Recurrence
}

\author{
Dependence on Tumor versus Tissue Microenvironment
}

Agnieszka K. Witkiewicz, ${ }^{\star \dagger \ddagger}$

Dayana B. Rivadeneira, ${ }^{\dagger \S}$ Adam Ertel, ${ }^{\dagger \S}$

Jessica Kline, * Terry Hyslop,"

Gordon F. Schwartz, ${ }^{\dagger \star *}$ Paolo Fortina, ${ }^{\dagger \S}$

and Erik S. Knudsen ${ }^{\dagger \S}$

From the Department of Pathology, Kimmel Cancer Center, ${ }^{\dagger}$

Breast Center, ${ }^{\ddagger}$ Department of Cancer Biology, ${ }^{\S}$ Cancer Genomics

Unit, ${ }^{\text {" }}$ and Departments of Pharmacology and Experimental

Therapeutics," and Surgery, ${ }^{* *}$ Thomas Jefferson University,

Philadelphia, Pennsylvania

The prevalence of ductal carcinoma in situ (DCIS) diagnoses has significantly increased as a result of active radiographic screening. Surgical resection and hormone and radiation therapies are effective treatments, but not all DCIS will progress to invasive breast cancer. Therefore, markers are needed to define tumors at low risk of recurrence and progression that can be treated by surgery alone rather than by adjuvant therapies. Initial analyses indicate that retinoblastoma (RB)-pathway perturbations occur at high frequency in DCIS and mirror the molecular alterations observed in invasive breast cancer. Particularly, the elevated expression of p16ink4a in DCIS was associated with loss of RB function and estrogen receptor-negative biology. Furthermore, high expression of p16ink $4 \mathrm{a}$ in conjunction with $\mathrm{Ki}-67 \mathrm{was}$ associated with increased risk of DCIS recurrence and progression to invasive disease in multivariate analyses. These data are consistent with a functional role for RB in modulating the invasive behavior of mammary epithelial cells. The tissue microenvironment is particularly relevant to the behavior of DCIS, and, surprisingly, elevated expression of p16ink4a in nonproliferative stroma was observed in a substantial fraction of cases. In this tissue compartment, p16ink4a expression was strongly associated with disease recurrence, independent of standard histopathologic features. Together, the data herein describe dual aspects of RB-pathway biology that are associated with disease recurrence through the epithelial or stromal compartment of DCIS. (Am J Pathol 2011, 179:1171-1178; DOI: 10.1016/j.ajpath.2011.05.043)

Breast cancer is the second most common cause of female cancer death and affects $\sim 10 \%$ of women in the western world. Increased screening for breast cancer by radiologic methods has had a dramatic effect on the number of detected cases and stage at diagnosis. In particular, more women are diagnosed with ductal carcinoma in situ (DCIS) lesions, ${ }^{1-3}$ a non-obligate precursor lesion to invasive disease, that has many biological features in common with breast cancer [eg, estrogen receptor (ER) and Her2 status]. ${ }^{1,3}$ Although $\sim 64,000$ cases of DCIS are diagnosed annually in the United States, the optimal care of such patients remains a significant challenge. ${ }^{1,4}$ Surgical resection is the primary therapy, but adjuvant radiotherapy and hormone therapy are commonly used with potential side effects and limited evidence for improved overall survival. ${ }^{5}$ Importantly, data have emerged that the retinoblastoma (RB) tumor suppressor pathway is a determinant in predicting the risk of recurrence in DCIS. ${ }^{6}$

The RB tumor suppressor is a key regulator of proliferation that is functionally perturbed in human cancer. ${ }^{7,8}$ Under normal physiological conditions, RB assembles transcriptional repression complexes that inhibit the expression of a program of genes that are required for cellular proliferation. ${ }^{9,10}$ The repressive activity of RB is alleviated in proliferating cells by the action of CDK/cyclin complexes that phosphorylate RB. In particular, CDK4/

Supported by NIHKOMEN.

Accepted for publication May 31, 2011.

A guest editor acted as Editor-in-Chief for this manuscript. No person at Thomas Jefferson University or Albert Einstein College of Medicine was involved in the peer review process or final disposition for this article.

Address reprint requests to Erik S. Knudsen, Ph.D., Department of Cancer Biology, Thomas Jefferson University, Philadelphia, PA 19107. E-mail: eknudsen@kimmelcancercenter.org. 
cyclin D complexes are rate limiting for the initiation of RB inactivation. However, the latent oncogenic activity of CDK4 and cyclin D1 is antagonized by p16ink4a, which is encoded by the CDKN2A tumor suppressor gene. ${ }^{11-13}$ In total, RB, cyclin D1, and p16ink4a define a pathway that is functionally inactivated in most cancers. ${ }^{7,12,14} \mathrm{Al}$ though the RB pathway is disrupted frequently, there is heterogeneity in the behavior of tumors harboring different lesions in this pathway. For example, cyclin D1 overexpression is relatively common in ER-positive breast cancer, ${ }^{15,16}$ whereas loss of RB occurs more frequently in ER-negative breast cancer. ${ }^{17}$ The effect of p16ink4a in cancer is complex. Overproduction of p16ink4a can be associated with the induction of cellular senescence and the blockade of tumor development. ${ }^{18}$ In this context, p16ink4a and the induced senescence is believed to represent a barrier to disease progression, although senescent cells have been postulated to secrete factors that could be involved in disease progression. ${ }^{19,20} \mathrm{Al}$ though loss of p16ink4a is observed frequently in cancer and is believed to allow bypass from senescence, ${ }^{21}$ the overexpression of p16ink4a is observed in a number of cancers wherein the RB tumor suppressor has been inactivated. ${ }^{22-24}$ Thus, whereas high levels of p16ink4a could denote senescence, the elevated expression of p16ink4a in a hyperproliferative setting is believed to be indicative of loss of RB function.

Previous work has indicated that DCIS lesions harboring elevated expression of p16ink4a in conjunction with a high proliferative index are prone to postsurgical recurrence. ${ }^{6}$ These studies provided an impetus to delineate the influence of the RB pathway in DCIS lesions and the local tumor environment.

\section{Materials and Methods}

\section{Cell Culture and Cell Cycle Analyses}

RB-proficient and -deficient MCF10A models were developed and propagated as previously described. ${ }^{25}$ Cell cycle and immunoblotting analyses were performed as previously described. ${ }^{25}$

\section{Migration Assay}

MCF10A cells were seeded $\left(5 \times 10^{4}\right.$ cells $)$ on Boyden Chambers (Franklin Lakes, NJ; BioCoat 354578) under low serum conditions. Complete growth medium was added to the wells as the chemoattractant. Chambers were placed in wells containing complete medium. The cells of the lower surface of the membrane were stained by placing the chambers in the wells containing DAPI diluted in PBS. Cells were scored with a fluorescent microscope.

\section{Tissue Staining and Scoring}

Formalin-fixed, paraffin-embedded tissue sections were cut at $4 \mu \mathrm{m}$ and deparaffinized by standard techniques. Antigen retrieval was performed by heating the sections in $10 \mathrm{mmol} / \mathrm{L}$ citrate buffer $\mathrm{pH} 6.0$ for 50 minutes with the use of a pressure cooker. For p16ink4a, sections were incubated with mouse monoclonal antibody at 1:50 dilution (MTM Laboratories, Inc., Westborough, MA; catalog no. 9518) and for Ki-67 with rabbit monoclonal antibody at 1:600 dilution (AbCam, Cambridge, MA; catalog no. ab16667) for 60 minutes at room temperature. Primary RB antibody, (Thermoscientific; catalog no. MS-107-B) was applied to slides and incubated overnight at $4^{\circ} \mathrm{C}$ with the use of a 1: 50 dilution. The immune complexes were visualized with Mouse ABC (Vector Laboratories, Inc., Burlingame, CA) and the chromogenic substrate Dako Liquid DAB + Substrate-Chromogen Solution (Dako North America, Inc., Carpinteria, CA; catalog no. K3468; diaminobenzidine tetrahydrochloride) for 3 minutes. For p16ink4a the staining was graded as 0 (no cells staining), $1+$ (diffuse weak nuclear or cytoplasmic blush or $<25 \%$ cell showing strong staining), $2+$ ( $25 \%$ to $75 \%$ of cells with strong staining), and $3+$ ( $>75 \%$ cells showing strong staining). Score $3+$ was considered high expression. Ki-67 labeling index was determined by counting $\geq 500$ nuclei in areas of the section with the highest labeling rates and was considered high when $\geq 10 \%$ of tumor cells were stained. ${ }^{26}$ For RB, only nuclear labeling was analyzed. Cases were considered negative for RB when no neoplastic cell nuclei showed labeling in sections in which stromal cells and endothelial cells stained. The scoring was performed by an experienced staff pathologist and another observer who had no prior knowledge of the disease recurrence status, hormone receptor status, HER2 status, or patient age.

\section{Microarray RNA Extraction, Hybridization, and Normalization}

Formalin-fixed paraffin-embedded tissues were first deparaffinized with a xylene-based extraction, followed by ethanol dehydration. Tissue samples were then disrupted during an overnight incubation with a Proteinase $\mathrm{K}$ lysis buffer. Nucleic acids were bound to a glass fiber filter in the presence of a chaotropic salt under conditions that were optimized specifically for RNA recovery. Bound RNA was subjected to a series of wash steps to remove contaminating cellular components, and any residual DNA was digested by incubation with DNasel. A second round of Proteinase $\mathrm{K}$ digestion, followed by further wash steps, was done to improve the final purity of the RNA, which was then eluted from the glass fibers in a small volume of low-salt elution buffer. All RNA samples were analyzed for concentration, purity, and integrity with the use of spectrophotometric methods in combination with the Agilent Bioanalyzer (Agilent Technologies, Palo Alto, CA). Total RNA was amplified with the NuGEN WT-Ovation FFPE RNA Amplification System V2. cDNA was fragmented and labeled with the Encore cDNA Biotin Module. The resultant fragmented and labeled cDNA was added to the hybridization cocktail in accordance with the NuGEN guidelines for hybridization onto Almac GeneChip arrays (Almac Group, Craigavon, UK). After the hybridiza- 


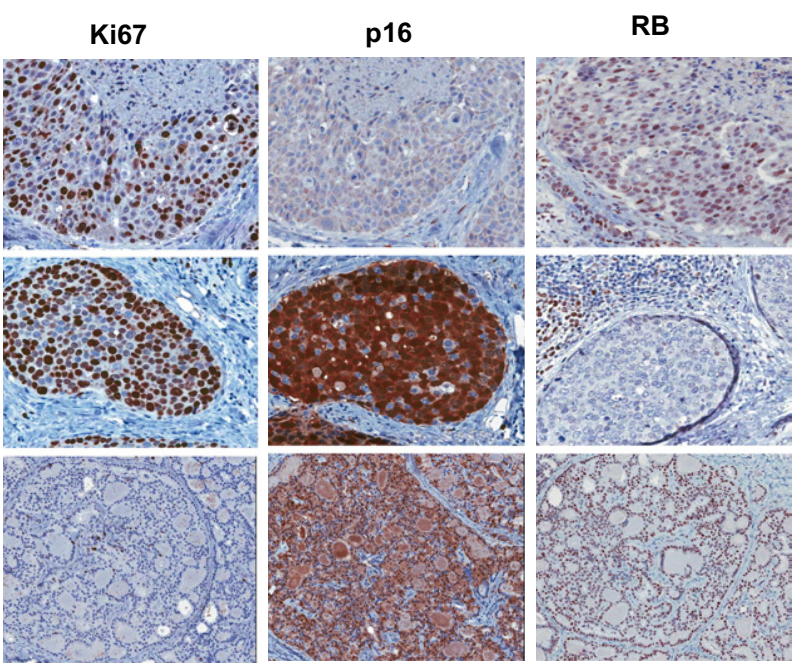

Figure 1. DCIS cases were stained for Ki-67 (left panels), p16ink4a (middle panels), and $\mathrm{RB}$ (right panels). Representative images of staining from three cases are shown. Original magnification: $\times 100$ (top and middle panels); $\times 50$ (bottom panels).

tion for 16 to 18 hours at $45^{\circ} \mathrm{C}$ in an Affymetrix GeneChip Hybridization Oven 640, the array was washed and stained on the GeneChip Fluidics Station 450 with the use of the appropriate fluidics script, before being inserted into the Affymetrix autoloader carousel and scanned with the GeneChip Scanner 3000. The Almac GeneChip array data were processed with the PLIER algorithm, including GC background correction and qutile normalization, in Affymetrix Expression Console version 1.1 (Affymetrix, Inc., Santa Clara, CA). Subsequent data processing and analyses were performed with MATLAB software (The Mathworks, Inc., Natick, MA). The PLIER + 16 expression scale was used because it has a high level of consistency with RT-PCR. ${ }^{27}$ To achieve this scale, data were offset by a value of 16 to achieve variance stabilization before log2 transformation.

\section{Differential Expression Analysis}

Differential expression of key cell cycle/Rb/p16 pathway genes and an RB loss expression signature was evaluated in comparisons between ER-positive versus ERnegative, and high-p16 [immunohistochemistry (IHC) score $3+$ ] versus low-p16 (IHC score of 0 to $2+$ ) samples. Significance for the differential expression of the $\mathrm{Rb} / \mathrm{p} 16$ pathway genes and an RB loss signature was determined with one-way analysis of variance. The RB loss expression signature, comprising 159 genes, was previously published. ${ }^{28}$ One hundred fifty-one of these genes are represented on the Almac custom breast cancer GeneChip. The average RB loss signature magnitude was computed by median centering expression profiles for each gene and then averaging over all 151 genes in the signature. This average signature magnitude was used to order samples along a gradient from low to high and to depict the expression of the RB loss signature along with ER status in an expression heat map.

\section{Comparison of Expression Profiles between DC/S and Infiltrating Ductal Carcinoma}

Pearson's correlation was computed among gene expression profiles to identify co-regulation among Rb/p16 pathway genes. An additional microarray data set representing infiltrating ductal carcinoma cases, compiled from the public databases and encompassing $>900$ samples from patients with breast cancer, ${ }^{29}$ was used to observe whether the same trends exist in DCIS versus infiltrating ductal carcinoma. Pearson's correlation was computed among the same gene expression profiles among this data set, and results from both data sets were depicted with the use of scatter plots.

A
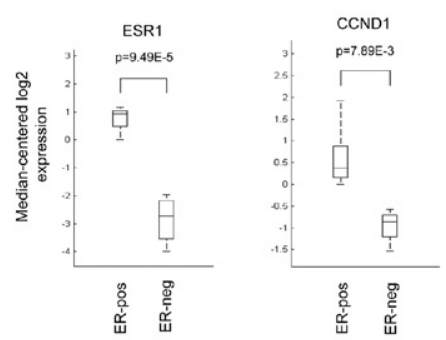

B
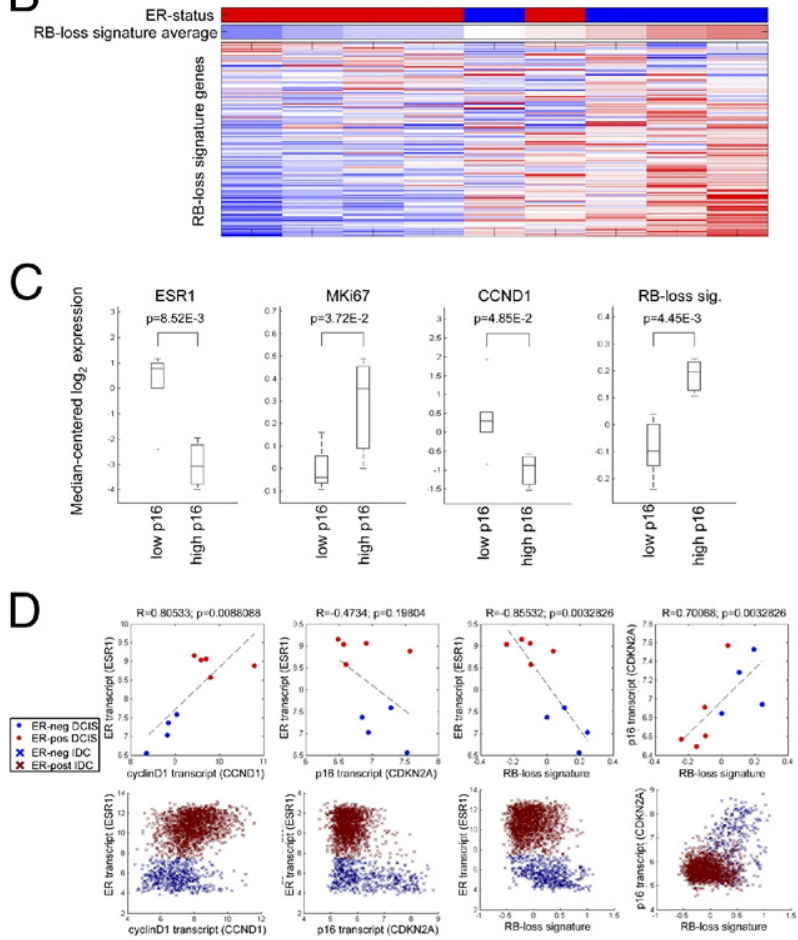

Figure 2. A: Microrray data from DCIS cases were stratified on the basis of ER status of DCIS cases, and normalized values of ER and cyclin D1 expression are shown. B: DCIS cases were clustered on the basis of the RB loss signature. The ER status and average RB loss signature are shown. C: The specific relation of p16ink4a tissue staining to ER, Ki-67, cyclin D1, and RB loss signature normalized expression levels are shown. D: Pairwise relations between RB pathway perturbations and ER status/p16ink4a are shown in DCIS (top panels) and invasive breast cancer (bottom panels). Red symbols denote ER-positive disease and blue symbols denote ER-negative disease. 


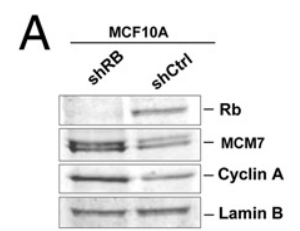

B
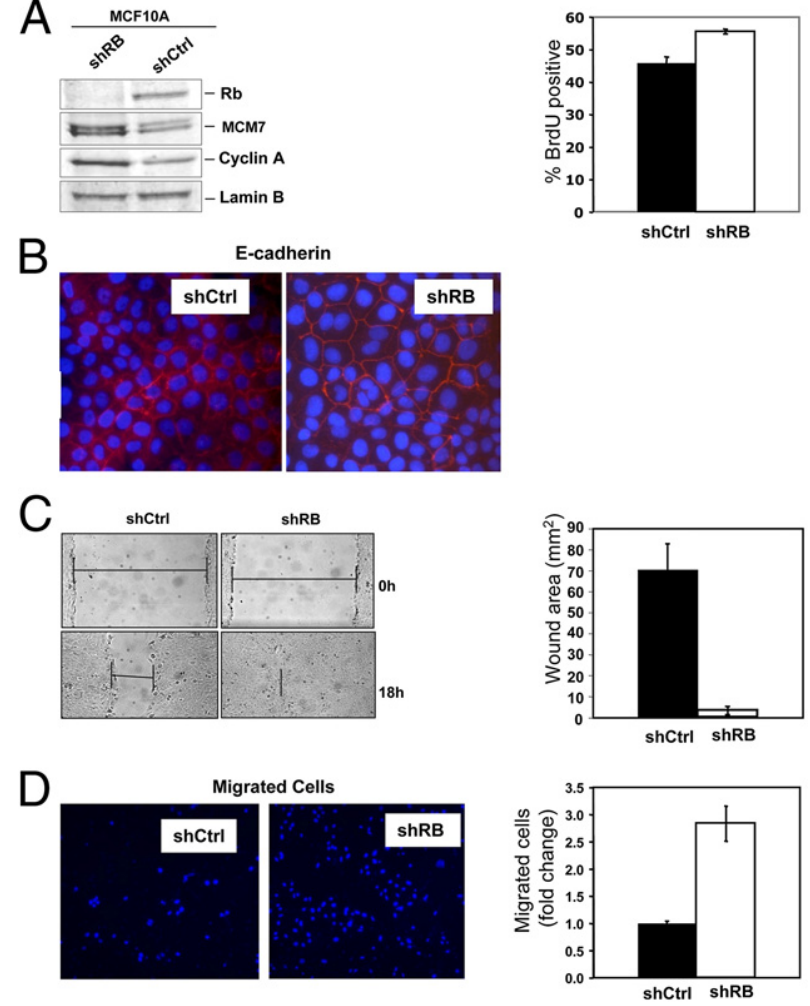

Figure 3. A: MCF10A cells were infected with retroviruses encoding shcontrol or sh-RB. Stable populations were subjected to immunoblotting with the indicated antibodies. MCF10A populations were labeled with bromodeoxyuridine, and the percentage of positive cells was determined. Data are from three independent experiments. B: MCF10A cells were stained for E-cadherin expression. Representative images are shown. C: Wound healing assays were performed on monolayers of MCF10A cells. The wound was measured at 24-hour intervals, and data presented are quantified from three independent experiments. D: The indicated cells populations were used to determine migration in a modified Boyden chamber assay; representative images of migrated cells are shown. The fold-difference in migration was quantified from three experiments. Sh, short hairpin.

\section{Statistical Analysis}

Univariate survival analysis was completed with KaplanMeier methods, and multivariable analysis was performed with Cox proportional hazards models. The association between age and stromal p16ink4a expression was analyzed with the Wilcoxon two-sample test. Prognostic variables, such as age, necrosis, and high nuclear grade, were left in the model regardless of significance. Biomarkers and their interactions were then added to the model and retained if significant at the 0.05 level. Cox models were assessed for the proportional assumption. Results are reported as adjusted hazards ratios, confidence intervals, and $P$ values. Computations were completed in $\mathrm{R}$.

\section{Results}

To determine the role of the RB pathway in DCIS, an investigation of the relation between pathway members was performed. Stainings for RB and p16ink4a were rigorously optimized, and coordinate staining of these markers was performed initially on $10 \mathrm{DCIS}$ cases, of which 3 cases are shown in Figure 1. These data showed several key relations. First, there is a clear dichotomy in p16ink4a staining. There are cases with low/absent p16ink4a expression, which retained the expression of RB (Figure 1, top row, $40 \%$ of cases). Interestingly, all cases with high p16ink4a levels ( $3+$ staining) were proliferative, as indicated by high Ki-67 index (Figure 1, middle row). In these lesions, there was also consistent lack of RB staining and this constituted $30 \%$ of cases. In contrast, lesions with moderate levels of p16ink4a and a low Ki-67 index exhibited nuclear RB staining and this constituted $10 \%$ of the cases (Figure 1, bottom row). These data indicate that high levels of p16ink4a in conjunction with a high proliferative index are indicative of RB functional loss.

To evaluate the features of RB-pathway dysregulation in relation to gene expression, microarray profiling was performed on microdissected DCIS specimens. For these analyses, we selected nine cases of DCIS for which the p16ink4a and RB status were determined (Figure 2). To confirm that the microarray analyses were consistent with the clinical assignment of the cases, ER expression from the profiled samples was compared against the histologic scoring. These validation analyses showed that tumors that were histologically ER positive exhibited elevated ER transcript levels (ESR1; Figure 2A). Thus, the microarray data have direct bearing on the biological origin of the DCIS lesion. These microarray data were then used to interrogate the overall dysregulation of RBpathway function in DCIS. In the DCIS lesions, there was a consistent correlation of cyclin D1 with ER-positive DCIS (CCND1; Figure 2A). This finding is highly reminis-

Table 1. Clinicopathologic Summary of the DCIS Cohort

\begin{tabular}{lc}
\hline & $N(\%)$ \\
\hline Age & \\
$<40$ yrs & $10(8)$ \\
40 to 60 yrs & $60(48)$ \\
$>60$ yrs & $56(44)$ \\
Recurrence & \\
Yes & $49(39)$ \\
No & $77(61)$ \\
Recurrence type & \\
DCIS & $33(67)$ \\
Invasive & $16(33)$ \\
Nuclear grade & \\
1 & $29(23)$ \\
2 & $57(45)$ \\
3 & $40(32)$ \\
Necrosis & \\
Present & $58(46)$ \\
Not present & $68(54)$ \\
Architectural type & \\
Comedo & $39(31)$ \\
Non-comedo & $87(69)$ \\
ER & $110(87)$ \\
Positive & $16(13)$ \\
Negative & \\
PR & $99(79)$ \\
Positive & $27(21)$ \\
Negative & $30(24)$ \\
Her2 & $96(76)$ \\
Positive & \\
Negative & \\
\hline
\end{tabular}

$\mathrm{PR}$, progesterone receptor. 
Table 2. Univariate Analyses of High p16ink4a/Ki67 Staining within the DCIS Lesion for the Cohort

\begin{tabular}{|c|c|c|c|}
\hline & \multicolumn{3}{|c|}{ High p16 and Ki67 } \\
\hline & $0, \mathrm{n}(\%)$ & $1, \mathrm{n}(\%)$ & $P$ \\
\hline \multicolumn{4}{|l|}{ PR } \\
\hline Negative & 7 (26) & $20(74)$ & \multirow[t]{2}{*}{$9.57 \times 10^{-5}$} \\
\hline Positive & $69(70)$ & $30(30)$ & \\
\hline \multicolumn{4}{|l|}{ ER } \\
\hline Negative & $2(12)$ & $14(88)$ & \multirow[t]{2}{*}{$3.99 \times 10^{-5}$} \\
\hline Positive & $74(67)$ & $36(33)$ & \\
\hline \multicolumn{4}{|l|}{ Her2 } \\
\hline Negative & $67(70)$ & $29(30)$ & \multirow[t]{2}{*}{0.000197} \\
\hline Positive & $9(30)$ & $21(70)$ & \\
\hline \multicolumn{4}{|l|}{ Necrosis } \\
\hline No & 47 (69) & $21(31)$ & \multirow[t]{2}{*}{0.044} \\
\hline Yes & $29(50)$ & $29(50)$ & \\
\hline \multicolumn{4}{|l|}{ ER-PR-Her2- } \\
\hline Yes & $0(0)$ & $7(100)$ & \multirow[t]{2}{*}{0.00118} \\
\hline No & $76(64)$ & $43(36)$ & \\
\hline \multicolumn{4}{|c|}{ Nuclear grade } \\
\hline Low (1) & $60(70)$ & $26(30)$ & \multirow[t]{2}{*}{0.00185} \\
\hline $\operatorname{High}(2,3)$ & $16(40)$ & $24(60)$ & \\
\hline \multicolumn{4}{|l|}{ Recurrence } \\
\hline No & $54(70)$ & $23(30)$ & \multirow[t]{2}{*}{0.00553} \\
\hline Yes & $22(45)$ & $27(55)$ & \\
\hline \multicolumn{4}{|c|}{ Bca recurrence } \\
\hline No & $54(70)$ & $23(30)$ & \multirow[t]{2}{*}{0.0205} \\
\hline Yes & $6(38)$ & $10(62)$ & \\
\hline \multicolumn{4}{|c|}{ DCIS recurrence } \\
\hline No & $54(70)$ & $23(30)$ & \multirow[t]{2}{*}{0.0505} \\
\hline Yes & $16(48)$ & $17(52)$ & \\
\hline
\end{tabular}

Bca, breast carcinoma; ER, estrogen receptor; PR, progesterone receptor.

cent of the known association of elevated cyclin D1 levels with ER-positive breast cancer. ${ }^{30,31}$ The RB transcript (Rb1) was largely unchanged across DCIS samples, indicating that the level of RB transcript levels are not a good prognosticator of RB protein expression (not shown). Therefore, to assess RB functional status, a gene expression signature of RB loss was used. This signature has been used in multiple studies to quantitatively assess the functionality of RB in tumor specimens, and it is largely consistent with the RB loss of heterozygosity signature defined by the laboratory of Dr. Charles $M$. Perou. ${ }^{22,28}$ The RB loss signature was used in hierarchical clustering of DCIS cases and showed an enrichment for ER-negative cases (Figure 2B). Correspondingly, with the use of the histologically determined levels of p16ink $4 a$ as a stratification criterion, it was found that DCIS lesions with elevated p16ink4a are generally ER negative, express high levels of the proliferation marker $\mathrm{Ki}-67$ with low cyclin D1 levels, and show RB loss signature (Figure $2 \mathrm{C}$ ). These findings indicate that DCIS lesions harbor distinct RB-pathway perturbations with a subset that harbors elevated p16ink4a and RB loss of function.

DCIS is viewed as a non-obligate precursor to invasive breast cancer. Therefore, it could be expected that alterations present in DCIS would be reflected in invasive disease. To interrogate this possibility RBpathway constituents were evaluated in an integrated cohort of ER-positive and -negative cases of breast cancer (Figure 2D). This cohort encompasses $>1000$ cases for which ER status is known. ${ }^{28}$ The analyses performed showed that the general deregulation of RB-pathway alterations observed in DCIS cases were conserved in invasive disease (Figure 2D). For example, ER-positive DCIS and invasive cancer both express higher levels of cyclin D1 (Figure 2D, left panels). Similarly, cases with

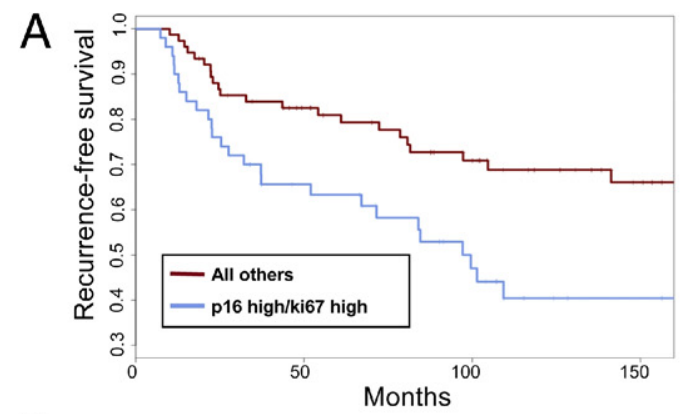

$\mathrm{B}$ p16-negative stroma p16-positive stroma
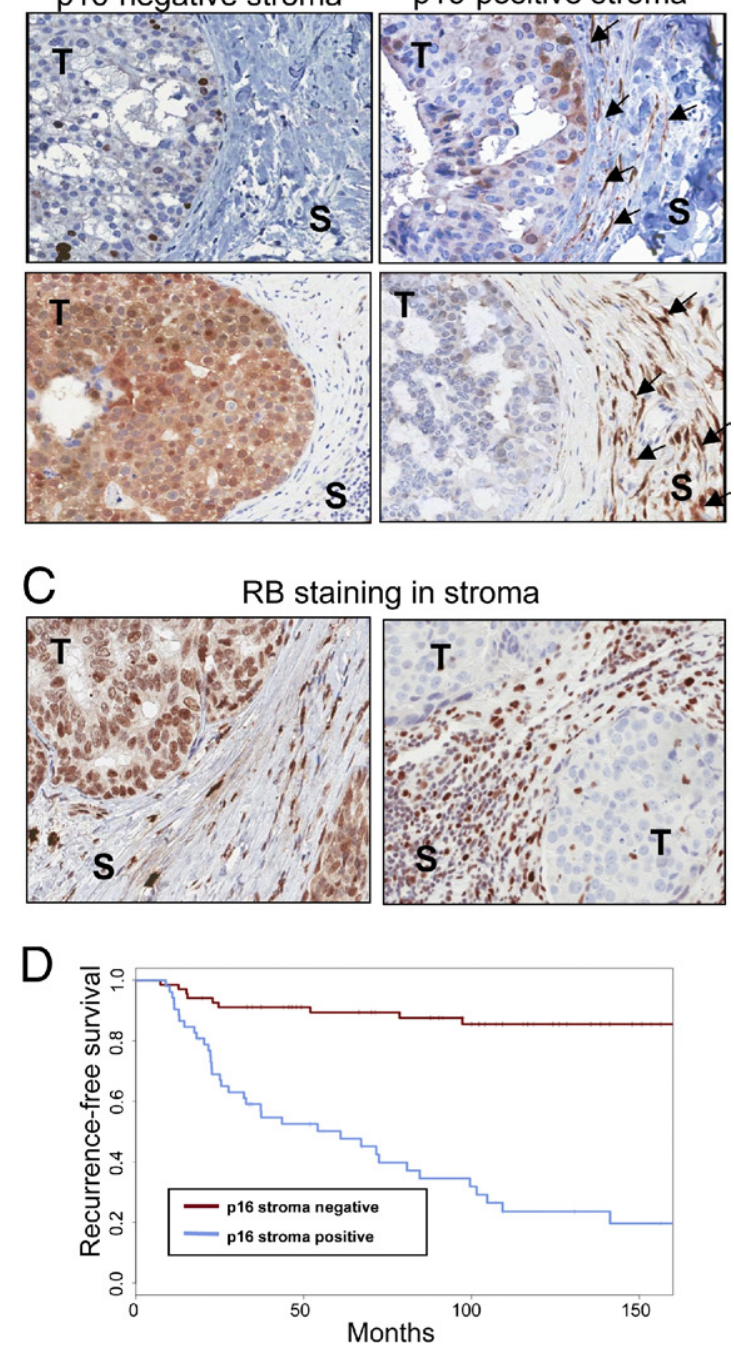

Figure 4. A: Kaplan-Meier analyses of disease recurrence on the basis of p16ink4a/Ki-67 in the DCIS lesions. B: Representative images of p16ink4a staining in the epithelial (T) and stromal compartment of DCIS (S). Stromal cells staining for p16ink $4 \mathrm{a}$ are indicated by arrows. C: Representative images of RB staining, showing the consistent expression of RB in the stroma (S). D: Kaplan-Meier analyses of disease recurrence on the basis of the stromal expression of p16ink4a. Original magnification: $\times 200(\mathbf{B}$ and C) 
Table 3. Multivariate Analyses of All Markers Analyzed in the DCIS Cohort

\begin{tabular}{lccc}
\hline & \multicolumn{3}{c}{ Cox model for time to recurrence } \\
\cline { 2 - 4 } & Hazard ratio & $95 \% \mathrm{Cl}$ & 0.546 \\
\hline Necrosis (yes versus no) & 0.84 & 0.47 to 1.49 & 0.027 \\
Nuclear grade (3 versus 1, 2) & 1.9 & 1.08 to 3.39 & 0.079 \\
Her2 (positive versus negative) & 1.75 & 0.94 to 3.3 & 0.456 \\
ER-PR-Her2 & 0.584 & 0.142 to 2.41 & 0.0283 \\
Year of diagnosis & 0.529 & 0.956 to 1 & 0.935 \\
Age & 0.978 & 1.24 to 4.77 & 0.010 \\
ki67 (high versus low) & 2.435 & 1.27 to 3.96 & 0.005 \\
p16 (high versus low) & 2.24 & 1.32 to 4.09 & 0.003 \\
p16 high ki67 high & 2.32 & 1.22 to 4.52 & 0.010 \\
p16 high ki67 high Her2 positive & 2.347 & 4.206 to 18.4 & $<0.0001$ \\
p16.stroma (positive versus negative) & 8.8 & & \\
\hline
\end{tabular}

$\mathrm{Cl}$, confidence interval; ER, estrogen receptor; PR, progesterone receptor.

high levels of p16ink4a and RB loss signature are generally ER negative (Figure 2D, middle panels). Finally, there is a positive correlation between high levels of p16ink4a and the RB loss signature (Figure 2D, right panels). These combined studies suggest that those alterations of the RB pathway observed in DCIS may play a role in subsequent tumor development.

To interrogate the functional effect of RB inactivation on the behavior of mammary epithelia, an immortalized model, MCF10A, was used (Figure 3). Derivatives of MCF10A produce DCIS-like lesions in xenograft mod-

Table 4. Univariate Analyses of High p16ink4a Staining in the Stroma in the DCIS Cohort

\begin{tabular}{|c|c|c|c|}
\hline & \multicolumn{3}{|c|}{ p16 stroma } \\
\hline & $0, \mathrm{n}(\%)$ & $1, \mathrm{n}(\%)$ & $P$ \\
\hline \multicolumn{4}{|l|}{ PR } \\
\hline Negative & $12(48)$ & $13(52)$ & 0.369 \\
\hline Positive & $56(59)$ & $39(41)$ & \\
\hline \multicolumn{4}{|l|}{ ER } \\
\hline Negative & $8(57)$ & $6(43)$ & 1 \\
\hline Positive & $60(57)$ & $46(43)$ & \\
\hline \multicolumn{4}{|l|}{ Her2 } \\
\hline Negative & $56(62)$ & $34(38)$ & 0.0544 \\
\hline Positive & $12(40)$ & $18(60)$ & \\
\hline \multicolumn{4}{|l|}{ Necrosis } \\
\hline No & $42(64)$ & $24(36)$ & 0.0988 \\
\hline Yes & $26(48)$ & 28 (52) & \\
\hline \multicolumn{4}{|l|}{ Age } \\
\hline Median & 59 & 54.5 & 0.39 \\
\hline \multicolumn{4}{|l|}{ ER-PR-Her2- } \\
\hline Yes & $3(60)$ & $2(40)$ & 1 \\
\hline No & $65(57)$ & $50(43)$ & \\
\hline \multicolumn{4}{|c|}{ Nuclear grade } \\
\hline Low (1) & $53(65)$ & $29(35)$ & 0.011 \\
\hline $\operatorname{High}(2,3)$ & $15(39)$ & $23(61)$ & \\
\hline \multicolumn{4}{|l|}{ Recurrence } \\
\hline No & $59(80)$ & $15(20)$ & $<0.0001$ \\
\hline Yes & 9 (20) & $37(80)$ & \\
\hline \multicolumn{4}{|c|}{ Bca recurrence } \\
\hline No & $59(80)$ & $15(20)$ & $<0.0001$ \\
\hline Yes & $1(6.2)$ & $15(94)$ & \\
\hline \multicolumn{4}{|c|}{ DCIS recurrence } \\
\hline No & $59(80)$ & $15(20)$ & $<0.0001$ \\
\hline Yes & $8(27)$ & $22(73)$ & \\
\hline
\end{tabular}
tor

Bca, breast cancer; ER, estrogen receptor; PR, progesterone recep- els ${ }^{32}$; therefore, such models could be informative about the biology of DCIS. With the use of stable short hairpin RNA expression, endogenous RB was efficiently knocked down in the MCF10A cells of the pooled populations analyzed (Figure $3 \mathrm{~A}$ ). The reduction of RB levels was associated with an increase in the expression of members of the signature for RB loss (eg, MCM7), and a modest but statistically significant increase in proliferative rate as measured by bromodeoxyuridine incorporation (Figure 3A). Importantly, RB knockdown did not compromise the epithelial nature of these cells, as indicated by E-cadherin staining (Figure 3B), and the ability to form acini in three-dimensional culture (data not shown). However, in wound healing assays performed in culture, RB deficiency resulted in increased invasive properties of the epithelial cells (Figure 3C). Quantitative assessment of cell migration further indicated that RB deficiency is associated with more invasive behavior of MCF10A cells (Figure 3D). These combined findings suggest that RB deficiency contributes to a more invasive epithelial phenotype that could contribute to recurrence.

To investigate the specific prognostic significance of RB-pathway disruption in DCIS, a unique patient cohort of 126 patients was used (clinicopathologic data are summarized in Table 1). All patients were treated by excision with 1-cm clear margin by a single surgeon and did not receive radiation or hormonal therapy. ${ }^{33} \mathrm{~A}$ total of 49 patients had recurrence, of which 16 recurred as invasive carcinoma and 33 recurred as DCIS. Expression of p16ink4a was determined by $\mathrm{IHC}$ and was generally associated with high $\mathrm{Ki}-67$ index $(P<0.001)$. The results of univariate association between high expression of p16ink4 and Ki-67 in DCIS epithelium ( $\mathrm{p} 16$ high and $\mathrm{Ki}-67$ high) and various clinicopathologic factors are shown in Table 2. Significant association was detected between high expression of p16ink4a and Ki-67 and high nuclear grade, presence of necrosis, and absence of ER/progesterone receptor (PR) expression. High expression of p16ink4a and Ki-67 was also associated with HER2-positive disease in both ER-positive and ER-negative cases $(P=0.012$ and $P<0.001$, respectively). Critically, the elevated expression of p16ink4a and Ki-67 was also associated with DCIS recurrence (Figure 4A). This association was true for both recurrence of DCIS and 
progression to invasive breast cancer (Table 2). In multivariate models, high p16ink4a expression in conjunction with high Ki-67 remained significant for recurrence as summarized in Table 3. In total, these findings suggest that RB-pathway dysregulation in the epithelial compartment is associated with a form of DCIS that is at increased risk of postsurgical recurrence and progression.

In performing analyses of p16ink4a staining, there was clear heterogeneity for intensity in the stroma surrounding DCIS (Figure 4B). Interestingly, this heterogeneity of p16ink4a in the stroma was not a reflection of p16ink4a levels in the DCIS lesion $(P=0.4)$. In addition, stromal p16ink4a expression was not associated with the ER, PR, or HER2 status of the DCIS lesion (Table 4). In the stroma there was no evidence for proliferation as indicated by Ki-67 staining (data not shown). In addition, in all cases the stroma stained positive for RB (Figure 4C). This combination of markers (ie, high p16ink4a, RB positive, and $\mathrm{Ki}-67$ low) is consistent with senescence occurring in the stromal environment of the DCIS lesion. Surprisingly, the elevated p16ink4a expression in the stroma was a strong predictor of disease recurrence in the DCIS (Figure 4D and Table 4). In multivariate models, stromal p16ink4a expression was an independent determinant of recurrence that exceeded any established marker interrogated in this cohort (Table 3 ).

\section{Discussion}

The relation of RB-pathway deregulation to breast cancer cause and progression is complex and related to stage of disease and tumor subtype. Although it has been previously reported that persons with heritable RB were at increased risk of breast cancer, ${ }^{34}$ only recently was this finding supported by animal models. ${ }^{35}$ As shown here, the RB pathway is compromised in DCIS lesions as detected by elevated p16ink4a expression. Although this finding has been previously described, ${ }^{6,24}$ here, we demonstrate that the p16ink4a-positive lesions that exhibit elevated Ki-67 are indeed deficient for RB protein, and such DCIS cases exhibit gene expression profiles indicative of RB loss. These findings suggest a significant frequency of RB loss or mutation in DCIS.

Provocative data suggest that RB-pathway dysregulation in DCIS and invasive cancer lesions can have a significant effect on clinical course. ${ }^{6}$ Particularly, the dysregulation of $\mathrm{p} 16 \mathrm{ink} 4 \mathrm{a}$ has been associated with an increased risk of recurrence and progression to invasive disease. The studies herein with the use of an independent patient cohort further support this conclusion. In our study the effect of p16ink4a, although most pronounced in ER-negative breast cancer, was also observed in other subtypes of disease. Functional studies indicate that RB loss can alter the invasive properties of mammary epithelia without directly altering the proliferative rate. ${ }^{36}$ This finding agrees well with the data from DCIS cases that p16ink4a staining provides prognostic information that goes beyond the proliferative index.

The effect of the tissue microenvironment on breast cancer pathogenesis is incontrovertible. Several studies indicate that alterations in cancer-associated fibroblasts can promote tumor growth and invasiveness by preventing cancer cell apoptosis, inducing cancer cell proliferation, and stimulating tumor angiogenesis. ${ }^{37-39}$ Here, we made the surprising observation that elevated p16ink4a is not only observed in the DCIS lesion but can also be observed in the stroma whose expression is independent of any of commonly measured markers in DCIS lesions. Unlike the DCIS lesions, the p16ink4a-positive stroma was uniformly devoid of Ki-67 and expressed RB. This combination of markers is consistent with the concept that such stromal cells are senescent. The signals responsible for inducing stromal fibroblast senescence presumably are derived from tumor cells, although stromal senescence might be an age-dependent process. The latter notion comes from observed accumulation of senescent fibroblasts in skin over time. ${ }^{40}$ However, no association was observed between stromal p16ink4a expression and patient age in our cohort. Furthermore, in staining multiple normal tissues, we failed to observe p16ink4a expression (data not shown). Together, these data suggest that stromal expression of p16ink4a is associated with a bidirectional communication between the DCIS lesion and the stroma and could be analogous to signals evoking senescence in response to oncogenic signals or DNA damage. ${ }^{41-43}$ It has been postulated that senescent stroma can contribute to disease progression by the secretion of cytokines and proteases, providing a mechanism through which the p16ink4a-positive stroma contributes to disease. ${ }^{19,20,44}$

In summary, these data show dual facets of the RB pathway related to the pathophysiology of DCIS: disruption of RB function within the DCIS lesion, as indicated by coordinated elevation of p16ink4a and Ki-67, is associated with increased risk of recurrence; and activation of the RB-pathway, as evidenced by elevated p16ink4a in nonproliferative stroma, is an independent determinant of recurrence.

\section{References}

1. Virnig BA, Tuttle TM, Shamliyan T, Kane RL: Ductal carcinoma in situ of the breast: a systematic review of incidence, treatment, and outcomes, J Natl Cancer Inst 2010, 102:170-178

2. Sakorafas GH, Farley DR, Peros G: Recent advances and current controversies in the management of DCIS of the breast. Cancer Treat Rev 2008, 34:483-497

3. Valenzuela M, Julian TB: Ductal carcinoma in situ: biology, diagnosis, and new therapies. Clin Breast Cancer 2007, 7:676-681

4. Patani N, Cutuli B, Mokbel K: Current management of DCIS: a review. Breast Cancer Res Treat 2008, 111:1-10

5. Houghton J, George WD, Cuzick J, Duggan C, Fentiman IS, Spittle M: Radiotherapy and tamoxifen in women with completely excised ductal carcinoma in situ of the breast in the UK, Australia, and New Zealand: randomised controlled trial, Lancet 2003, 362:95-102

6. Gauthier ML, Berman HK, Miller C, Kozakeiwicz K, Chew K, Moore D, Rabban J, Chen YY, Kerlikowske K, Tlsty TD: Abrogated response to cellular stress identifies DCIS associated with subsequent tumor events and defines basal-like breast tumors. Cancer Cell 2007, 12: 479-491

7. Burkhart DL, Sage J: Cellular mechanisms of tumour suppression by the retinoblastoma gene. Nat Rev Cancer 2008, 8:671-682

8. Knudsen ES, Knudsen KE: Tailoring to RB: tumour suppressor status and therapeutic response, Nat Rev Cancer 2008, 8:714-724 
9. Blais A, Dynlacht BD: E2F-associated chromatin modifiers and cell cycle control. Curr Opin Cell Biol 2007, 19:658-662

10. Nevins JR: E2F: a link between the Rb tumor suppressor protein and viral oncoproteins. Science 1992, 258:424-429

11. Arnold A, Papanikolaou A: Cyclin D1 in breast cancer pathogenesis. J Clin Oncol 2005, 23:4215-4224

12. Sherr CJ, McCormick F: The RB and p53 pathways in cancer. Cancer Cell 2002, 2:103-112

13. Bartkova J, Lukas J, Bartek J: Aberrations of the G1- and G1/S-regulating genes in human cancer. Prog Cell Cycle Res 1997, 3:211-220

14. Cobrinik D: Pocket proteins and cell cycle control. Oncogene 2005 , 24:2796-2809

15. van Diest PJ, Michalides RJ, Jannink L, van der ValkP, Peterse HL, de Jong JS, Meijer CJ, Baak JP: Cyclin D1 expression in invasive breast cancer. Correlations and prognostic value, Am J Pathol 1997, 150: 705-711

16. Loden M, Stighall M, Nielsen NH, Roos G, Emdin SO, Ostlund H, Landberg G: The cyclin D1 high and cyclin E high subgroups of breast cancer: separate pathways in tumorogenesis based on pattern of genetic aberrations and inactivation of the pRb node. Oncogene 2002, 21:4680-4690

17. Trere D, Brighenti E, Donati G, Ceccarelli C, Santini D, Taffurelli M, Montanaro L, Derenzini M: High prevalence of retinoblastoma protein loss in triple-negative breast cancers and its association with a good prognosis in patients treated with adjuvant chemotherapy. Ann Oncol 2009, 20:1818-1823

18. Bartkova J, Rezaei N, Liontos M, Karakaidos P, Kletsas D, Issaeva N, Vassiliou LV, Kolettas E, Niforou K, Zoumpourlis VC, Takaoka M, Nakagawa H, Tort F, Fugger K, Johansson F, Sehested M, Andersen CL, Dyrskjot L, Orntoft T, Lukas J, Kittas C, Helleday T, Halazonetis TD, Bartek J, Gorgoulis VG: Oncogene-induced senescence is part of the tumorigenesis barrier imposed by DNA damage checkpoints. Nature 2006, 444:633-637

19. Davalos AR, Coppe JP, Campisi J, Desprez PY: Senescent cells as a source of inflammatory factors for tumor progression, Cancer Metastasis Rev 2010, 29:273-283

20. Coppe JP, Desprez PY, Krtolica A, Campisi J: The senescenceassociated secretory phenotype: the dark side of tumor suppression, Annu Rev Pathol 2010, 5:99-118

21. Oliveira AM, Ross JS, Fletcher JA: Tumor suppressor genes in breast cancer: the gatekeepers and the caretakers, Am J Clin Pathol 2005, 124(Suppl):S16-S28

22. Herschkowitz JI, He X, Fan C, Perou CM: The functional loss of the retinoblastoma tumour suppressor is a common event in basal-like and luminal B breast carcinomas. Breast Cancer Res 2008, 10:R75

23. Hollestelle A, Nagel JH, Smid M, Lam S, Elstrodt F, Wasielewski M, Ng SS, French PJ, Peeters JK, Rozendaal MJ, Riaz M, Koopman DG, Ten Hagen TL, de Leeuw BH, Zwarthoff EC, Teunisse A, van der Spek PJ, Klijn JG, Dinjens WN, Ethier SP, Clevers H, Jochemsen AG, den Bakker MA, Foekens JA, Martens JW, Schutte M: Distinct gene mutation profiles among luminal-type and basal-type breast cancer cell lines. Breast Cancer Res Treat 2010, 121:53-64

24. Subhawong AP, Subhawong T, Nassar H, Kouprina N, Begum S Vang R, Westra WH, Argani P: Most basal-like breast carcinomas demonstrate the same Rb-/p16+ immunophenotype as the HPVrelated poorly differentiated squamous cell carcinomas which they resemble morphologically. Am J Surg Pathol 2009, 33:163-175

25. Dean JL, Thangavel C, McClendon AK, Reed CA, Knudsen ES: Therapeutic CDK4/6 inhibition in breast cancer: key mechanisms of response and failure, Oncogene 2010, 29:4018-4032

26. Kerlikowske K, Molinaro AM, Gauthier ML, Berman HK, Waldman F, Bennington J, Sanchez H, Jimenez C, Stewart K, Chew K, Ljung BM, TIsty TD: Biomarker expression and risk of subsequent tumors after initial ductal carcinoma in situ diagnosis, J Natl Cancer Inst 2010, 102:627-637

27. Gyorffy B, Molnar B, Lage H, Szallasi Z, Eklund AC: Evaluation of microarray preprocessing algorithms based on concordance with RT-PCR in clinical samples. PLoS One 2009, 4:e5645

28. Ertel A, Dean JL, Rui H, Liu C, Witkiewicz AK, Knudsen KE, Knudsen ES: RB-pathway disruption in breast cancer: differential association with disease subtypes, disease-specific prognosis and therapeutic response. Cell Cycle 2010, 9:4153-4163

29. Ertel A, Dean JL, Rui H, Liu C, Witkiewicz A, Knudsen KE, Knudsen ES: RB-pathway disruption in breast cancer: differential association with disease subtypes, disease-specific prognosis, and therapeutic response. Cell Cycle 2010, 9:4153-4163

30. Nielsen NH, Emdin SO, Cajander J, Landberg G: Deregulation of cyclin E and D1 in breast cancer is associated with inactivation of the retinoblastoma protein. Oncogene 1997, 14:295-304

31. Millar EK, Dean JL, McNeil CM, O'Toole SA, Henshall SM, Tran T, Lin J, Quong A, Comstock CE, Witkiewicz A, Musgrove EA, Rui H, Lemarchand L, Setiawan VW, Haiman CA, Knudsen KE, Sutherland RL, Knudsen ES: Cyclin D1b protein expression in breast cancer is independent of cyclin D1a and associated with poor disease outcome. Oncogene 2009, 28:1812-1820

32. Behbod F, Kittrell FS, LaMarca H, Edwards D, Kerbawy S, Heestand JC, Young E, Mukhopadhyay P, Yeh HW, Allred DC, Hu M, Polyak K, Rosen JM, Medina D: An intraductal human-in-mouse transplantation model mimics the subtypes of ductal carcinoma in situ. Breast Cancer Res 2009, 11:R66

33. Witkiewicz AK, Dasgupta A, Nguyen KH, Liu C, Kovatich AJ, Schwartz GF, Pestell RG, Sotgia F, Rui H, Lisanti MP: Stromal caveolin-1 levels predict early DCIS progression to invasive breast cancer. Cancer Biol Ther 2009, 8:1071-1079

34. Kaye FJ, Harbour JW: For whom the bell tolls: susceptibility to common adult cancers in retinoblastoma survivors. J Natl Cancer Inst 2004, 96:342-343

35. Jiang Z, Deng T, Jones R, Li H, Herschkowitz JI, Liu JC, Weigman VJ, Tsao MS, Lane TF, Perou CM, Zacksenhaus E: Rb deletion in mouse mammary progenitors induces luminal-B or basal-like/EMT tumor subtypes depending on p53 status, J Clin Invest 2010, 120:32963309

36. Arima $Y$, Inoue $Y$, Shibata $T$, Hayashi $H$, Nagano O, Saya $H$, Taya $Y$ : $\mathrm{Rb}$ depletion results in deregulation of $\mathrm{E}$-cadherin and induction of cellular phenotypic changes that are characteristic of the epithelialto-mesenchymal transition. Cancer Res 2008, 68:5104-5112

37. Mueller MM, Fusenig NE: Friends or foes - bipolar effects of the tumour stroma in cancer. Nat Rev Cancer 2004, 4:839-849

38. Bhowmick NA, Neilson EG, Moses HL: Stromal fibroblasts in cancer initiation and progression. Nature 2004, 432:332-337

39. Kalluri R, Zeisberg M: Fibroblasts in cancer. Nat Rev Cancer 2006 , 6:392-401

40. Ressler S, Bartkova J, Niederegger H, Bartek J, ScharffetterKochanek K, Jansen-Durr P, Wlaschek M: p16INK4A is a robust in vivo biomarker of cellular aging in human skin. Aging Cell 2006, 5:379-389

41. Bartkova J, Horejsi Z, Koed K, Kramer A, Tort F, Zieger K, Guldberg P, Sehested M, Nesland JM, Lukas C, Orntoft T, Lukas J, Bartek J: DNA damage response as a candidate anti-cancer barrier in early human tumorigenesis. Nature 2005, 434:864-870

42. Bartek J, Bartkova J, Lukas J: DNA damage signalling guards against activated oncogenes and tumour progression. Oncogene 2007, 26: 7773-7779

43. Serrano M, Blasco MA: Putting the stress on senescence. Curr Opin Cell Biol 2001, 13:748-753

44. Burton DG: Cellular senescence, ageing and disease. Age (Dordr) 2009, 31:1-9 